\title{
Smartphone-Based Colorimetric Method to Quantify Iron Concentration and to Determine the Nanoparticle Size from Suspensions of Magnetic Nanoparticles
}

Yilian Fernández-Afonso, Gorka Salas, Irene Fernández-Barahona, Fernando Herranz, Cordula Grüttner, Jesús Martínez de la Fuente, María del Puerto Morales, Lucía Gutiérrez*

Y. Fernández-Afonso, Dr. L. Gutiérrez Department of Analytical Chemistry, Instituto Universitario de Nanociencia de Aragón (INA), Universidad de Zaragoza, 50018 Zaragoza, Spain

E-mail: lu@unizar.es

Y. Fernández-Afonso, Prof. J. M. de la Fuente, Dr. L. Gutiérrez

Instituto de Ciencia de Materiales de Aragón (ICMA), 50018 Zaragoza, Spain

Dr. G. Salas

IMDEA Nanociencia, 28049 Madrid, Spain

I. Fernández-Barahona, Dr. F. Herranz

Instituto de Química Médica, Consejo Superior de Investigaciones Científicas (CSIC), 28006 Madrid, Spain

I. Fernández-Barahona

Facultad de Farmacia, Universidad Complutense de Madrid, Madrid, Spain.

Dr. F. Herranz

Centro de Investigación Biomédica en Red de Enfermedades Respiratorias (CIBERES), Spain

Dr. C. Grüttner

Micromod Partikeltechnologie GmbH, D-18119 Rostock, Germany

Prof. J. M. de la Fuente, Dr. L. Gutiérrez

Centro de Investigación Biomédica en Red de Bioingeniería, Biomateriales y Nanomedicina (CIBER-BBN), Spain

Prof. M. P. Morales

Department of Energy, Environment and Health, Instituto de Ciencia de Materiales de Madrid (ICMM-CSIC), 28049 Madrid, Spain

Keywords: iron oxides, magnetic nanoparticles, iron quantification, particle size, smartphones, colorimetric methods

\section{Abstract}

Advanced uses of smartphones are changing our lifestyle and may have a great impact in materials sciences in the near future. In this work, the use of these devices to develop fast, 


\section{WILEY-VCH}

simple and cheap methods to characterize magnetic nanoparticle suspensions is tested. A series of dilutions of a wide library of magnetic nanoparticles, composed of iron oxide materials in the range between 3 and $43 \mathrm{~nm}$, with two different shapes and four different coatings has been prepared. The colloid color has been analyzed using the RGB (Red, Green, Blue) color model. Ratios of these parameters have been correlated with the suspension iron concentration and with the nanoparticles average size. A linear relationship between the color (in particular the $\mathrm{G} / \mathrm{R}$ ratio) and both the colloid iron content and the particles size has been found. The link between these parameters allows the development of two new methods to determine either the concentration or the particle size of magnetic nanoparticle suspensions just by acquiring images from suspensions of iron oxide magnetic nanoparticles with a smartphone.

\section{Introduction}

The ubiquity of smartphones and the fast development of different mobile apps is having a great impact in our daily lifestyle. Advanced applications of such devices are being currently developed in different scientific areas. Some examples of smartphone applications for scientific purposes include the collection of data in plant biology ${ }^{[1]}$ and the detection of heavy metal contamination in ecology studies. ${ }^{[2]}$ Nevertheless, to date, smartphones have been scarcely applied to material sciences and their application in this research field may provide a huge leap in terms of routine sample analysis with a very accessible, simple, fast, portable and low-cost strategy.

Smartphone cameras acquire images using a red, green, and blue (RGB) color code. Based on this code, several apps allow the easy analysis of these digital images to quantify color changes. These changes in color have been used for the determination of different analytes in liquid suspensions, both in simple solutions and direct sampling matrices such as blood or ground water. ${ }^{[3]}$ Several studies have also used smartphone analysis of color to improve the accuracy of the analysis of colorimetric diagnostic assays. ${ }^{[4-5]}$ In many cases, a reagent (either a molecule 


\section{WILEY-VCH}

or a nanoparticle) is used to produce a colored compound through the reaction with a particular analyte, and it is the color of the newly formed analyte-reagent complex what is detected. Furthermore, several smartphone accessories have been developed to allow the easy quantification of these analytes. ${ }^{[6]}$ In general, all these approaches improve the accuracy of the color analysis and are an excellent tool for the analysis of several substances either in remote areas ${ }^{[7]}$ or as part of POC (point-of-care) testing. ${ }^{[8]}$

The main objective of our work is the evaluation of RGB data acquired from images taken with smartphones to analyze nanomaterials. In particular, we have studied suspensions of iron oxide magnetic nanoparticles (NPs). In the frame of biomedical applications, iron oxides (mainly magnetite and maghemite) have raised a special interest given their low toxicity and their interesting magnetic properties, showing a great potential to revolutionize classical medical treatments. ${ }^{[9]}$ Suspensions of these iron oxide magnetic nanoparticles present colors that go from black, for concentrated samples, to yellowish, for the diluted ones. Both absorption and scattering processes are responsible for these changes. ${ }^{[10]}$

Our work has had two main purposes. The first one is the determination of the iron concentration from the color of the nanoparticle suspensions. This is a relatively direct approach similar to other proposed methods for the quantification of different analytes in which an unknown RGB value will be used to interpolate the data on a previously determined calibration curve. The second purpose is the development of a novel, and not so straightforward, approach to determine the nanoparticle size from the suspensions' color. The relationship between the nanoparticle size and the color of the suspensions has allowed us to estimate the nanoparticle core size, provided that the iron concentration of the nanoparticle suspensions is known.

\section{Results and Discussion}

\subsection{Materials tested}




\section{WILEY-VCH}

A library of magnetic nanoparticles has been prepared (Figure 1 and Figure S1 of the supporting information). Ten different sets of magnetic nanoparticles covering a wide range of sizes (3-43 nm) have been selected. Materials have been chosen in order to cover different sizes, shapes and nanoparticle coatings (Table S1 of the supporting information).

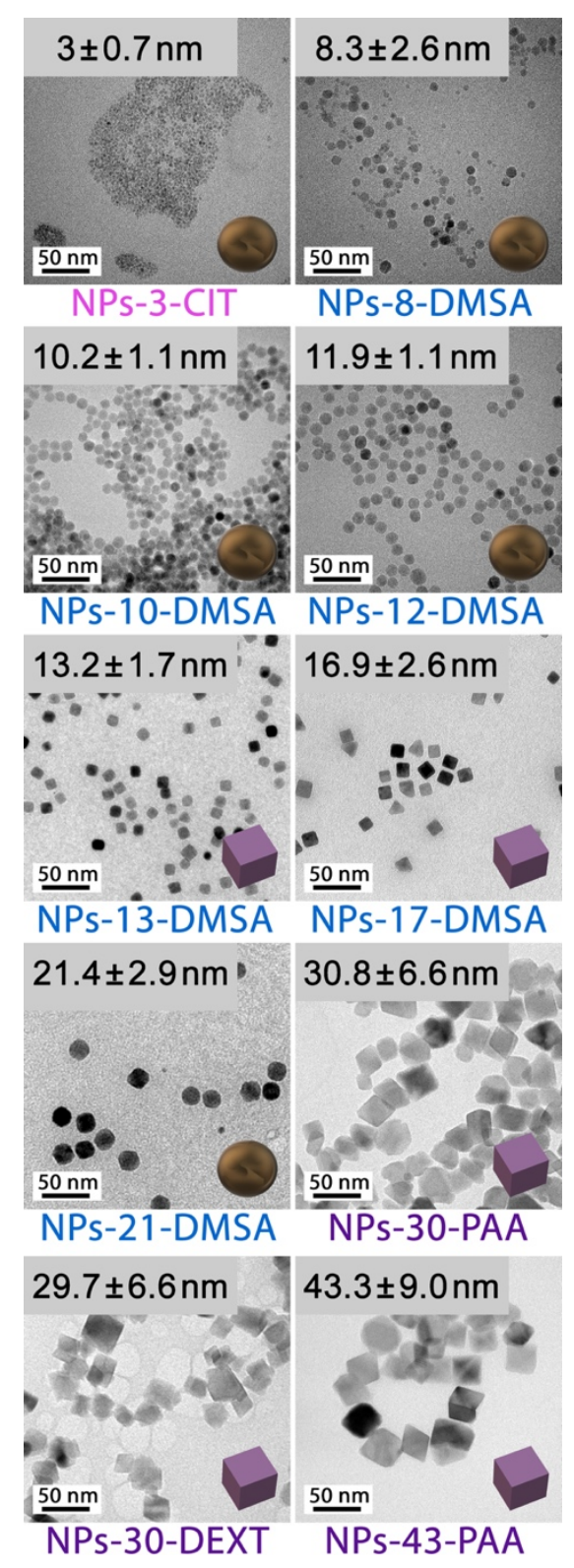

Figure 1. Transmission electron microscopy images at the same magnification for all the tested magnetic nanoparticles (Scale bar $=50 \mathrm{~nm}$ ). NPs have been grouped depending on synthesis protocol used for their preparation (Color code of the names: pink $=$ microwave assisted coprecipitation, blue $=$ thermal decomposition and purple $=$ oxidative precipitation). The 


\section{WILEY-VCH}

average size and shape of the particles are indicated at the top left and bottom right part of each image respectively. The name of each material includes the average core size and the nanoparticles coating (Coating codes: CIT $=$ citric acid, DMSA $=$ dimercaptosuccinic acid, $\mathrm{PAA}=$ polyacrylic acid and DEXT $=$ dextran $)$.

Only ferrimagnetic iron oxides (either magnetite, maghemite, or intermediate compositions along the oxidation of magnetite into maghemite ${ }^{[11]}$ have been considered for this analysis, as these are the most commonly used materials in the frame of biomedical applications.

Several synthesis routes have been followed in order to achieve such a wide range of sizes and shapes (Figure 1 and Table S1 of the supporting information). Microwave assisted coprecipitation has been used to obtain the smallest particles of $\approx 3 \mathrm{~nm}$ of average core size. Thermal decomposition of metalorganic precursors has been selected for the production of six different particles in the middle size range (between $\approx 8$ and $\approx 21 \mathrm{~nm}$ ). For this series, all the particles with different sizes have been coated with meso-2,3-dimercaptosuccinic acid (DMSA). Four of them are spherical while the other two present cubic morphology. Finally, oxidative precipitation has been used to produce the particles in the upper size range $(\approx 30-43 \mathrm{~nm})$ rendering cubic particles.

The main strengths of this library of magnetic NPs are that it covers a wide range of particle sizes, it contains particles with two different shapes, it has particles with the same coating but different sizes and also particles of the same size and different coatings. All these combinations are key parameters to evaluate the effect of the size, shape and coating on the colorimetric properties of the suspensions. The only concern about our library could be the possible transformations on the composition of our particles with time and its associated impact with their color. Magnetite is generally described as a black mineral while maghemite is red-brown one. ${ }^{[12]}$ It is generally accepted that, water suspensions of magnetite nanoparticles evolve to 


\section{WILEY-VCH}

maghemite with time. As all the NP suspensions studied in this work have been stored in water for relatively long times after their synthesis (months), it can be assumed that all of our particles contain, at least, an external layer of maghemite. In fact, all the characterized suspensions presented the typical brown color of maghemite. Therefore, it could be assumed that no further transformations on the crystalline structure have occurred during the characterization experiments.

\subsection{RGB data acquisition protocol}

With the purpose of assessing the concentration range in which the RGB determination is valid, samples have been diluted from the highest concentration available (at least $\approx 2 \mathrm{mg} \mathrm{Fe} \mathrm{mL}^{-1}$ for

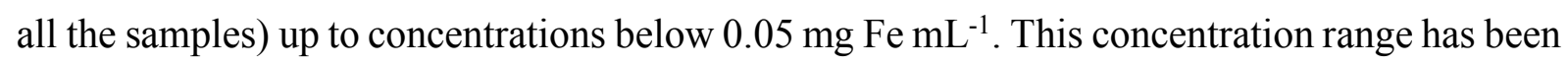
selected as it is the one in which clear differences in color are easily observed visually, going from black for the highest concentrations to colorless for the most diluted ones. This range is also especially interesting as it covers the concentrations generally used in routine laboratory procedures, such as physico-chemical characterization, ${ }^{[13]}$ functionalization protocols, ${ }^{[14]}$ in vitro procedures (e.g. toxicity and viability tests) ${ }^{[15-16]}$ or in vivo administration of the nanoparticles. ${ }^{[17-18]}$

One of the main difficulties of the image acquisition with the smartphone is the effect of the lighting conditions in the color determination reproducibility. ${ }^{[4-19]}$ To solve this problem and assure that the effects from the surrounding light are minimized we have used a closed carboard box to place the different suspensions of particles inside it (Figure 2 A). A hole on the upper side of the box has been made to place the camera always at the same distance from the sample $(5 \mathrm{~cm})$. This hole also allows the use of the flashlight from the smartphone to keep the lightning conditions constant. This box is bigger than the in 96-well plate used to place the samples, allowing the location of the smartphone camera always on top of the well where the RGB data 


\section{WILEY-VCH}

are acquired. Images from the different solutions have been acquired using the ColorGrab app (v 3.6.1 Loomatix Ltd.) that provides the RGB values coding the color for each suspension at the lightning conditions during the image acquisition. The range of the RGB values goes from $(0,0,0)$, for the black most concentrated samples, to $(255,255,255)$ for the colorless ones in which the image is white due to the background color below the nanoparticle suspensions (Figure 2A).
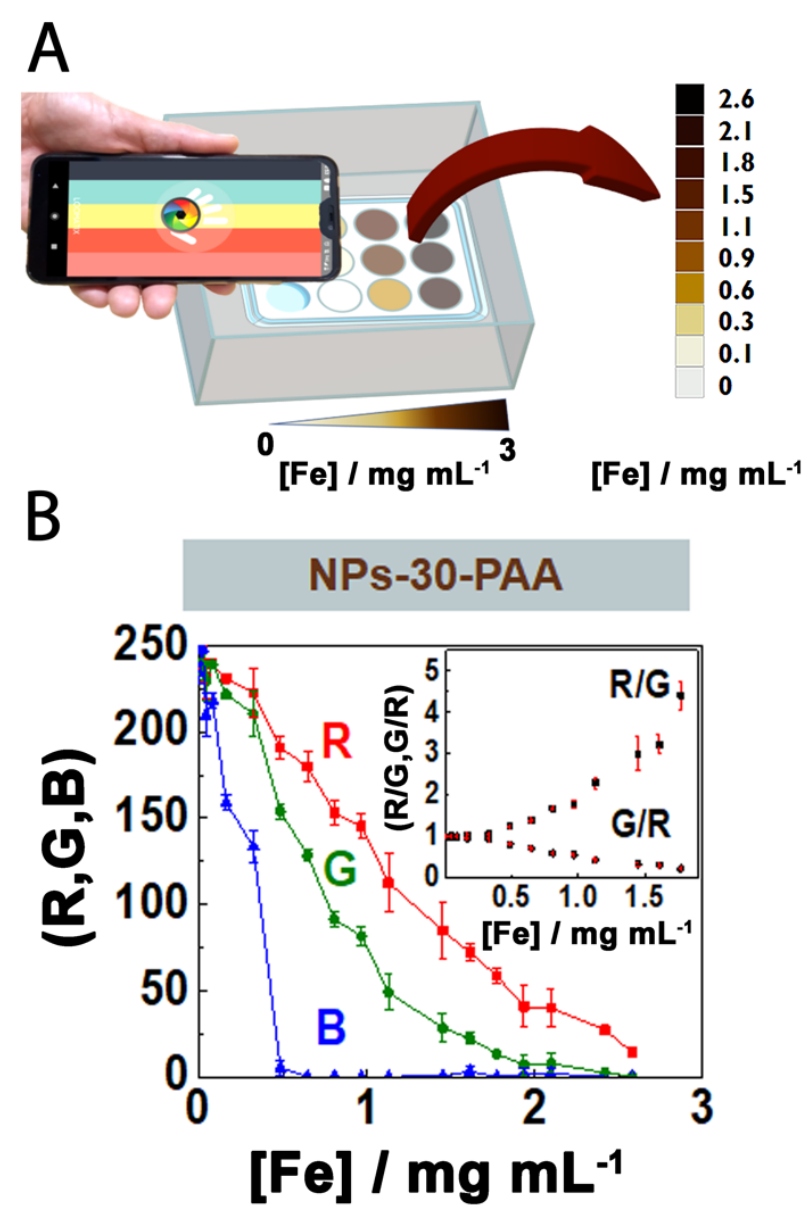

Figure 2. (A) Schematic representation of the image acquisition procedure. A closed box with an upper hole is used to maintain the distance between the smartphone and the samples. This design also allows maintaining the lightning conditions. The color acquired for suspensions of sample NPs-30-PAA at different iron concentrations is shown. (B) R, G and B values from images acquired from suspensions of sample NPs-30-DEXT prepared with different iron concentrations; (inset) Ratio of R/G and G/R from the same dilution series. 


\section{WILEY-VCH}

\subsection{RGB data could be used to determine the iron concentration in nanoparticle}

suspensions

The $R, G$ and $B$ values acquired for a dilution series of NPs-30 PAA have been plotted as a function of the iron concentration in Figure 2B (the rest of the nanoparticles are shown in Figure S2 from the supporting information). The R, G and B values at different concentrations display an interesting feature: B values saturate at significantly lower concentrations $(0.5 \mathrm{mg}$ Fe $\mathrm{mL}^{-1}$ ) than $\mathrm{G}$ and $\mathrm{R}$ values ( 2 and $3 \mathrm{mg} \mathrm{Fe} \mathrm{mL}^{-1}$ respectively). Therefore, further analysis of the ratio of these two variables ( $\mathrm{G}$ and $\mathrm{R}$ ) has been performed in order to improve the correlation between color and iron concentration (Figure 2B, inset). We have found that, within a given concentration range, the $\mathrm{G} / \mathrm{R}$ ratio displays a linear correlation with the iron concentration for all of the nanoparticle suspensions evaluated in this work (Figure 2B inset and S3 from the supporting information). In particular, the range in which this dependence is linear with the concentration is broader for the $\mathrm{G} / \mathrm{R}$ ratio than for the $\mathrm{R} / \mathrm{G}$ one. Therefore, this ratio is the one that has been selected for the rest of the data analysis presented this work.

The reproducibility of the method has been verified by several experiments (Figure 3). Multiple images of a single sample and images from different suspensions ( 3 identical samples for each dilution point) have been compared to evaluate the variability on the RGB data (Figure 3A). Also, measurements of the whole dilution series have been acquired in different days (Figure 3B). Moreover, smartphones from different brands have been used to compare the acquired RGB data of a series of dilutions of two representative samples (NPs-10-DMSA and NPs-30PAA) (Figure 3C). In all cases the deviations observed are minimal. Furthermore, RGB data of all the analyzed samples have been acquired several times along $1 \mathrm{~h}$ to verify that no changes were observed due to aggregation processes that could have an undesired impact on the suspension color (Figure 3D). All these measurements have assured the reproducibility of the experimental results. 


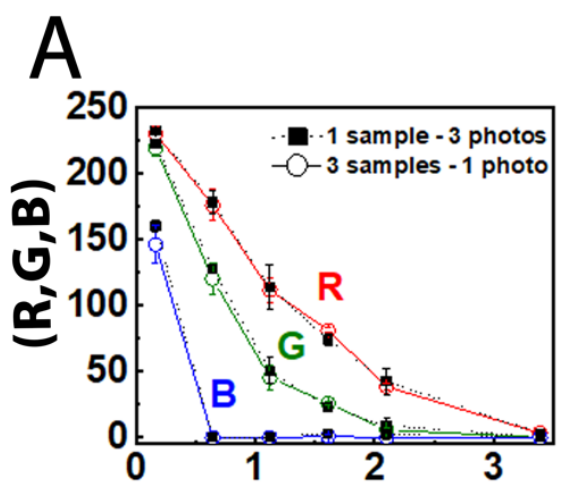

B

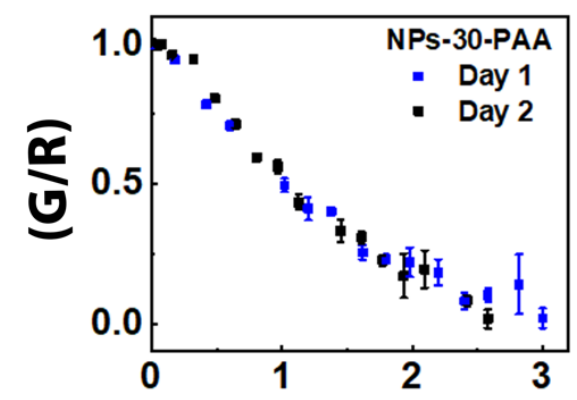

[Fe] $/ \mathrm{mg} \mathrm{mL}^{-1}$
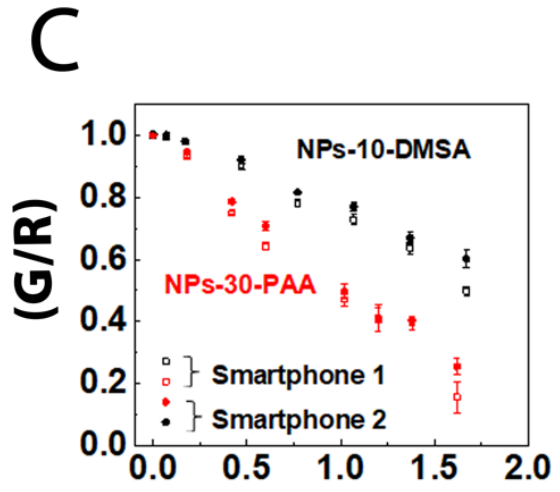

[Fe] / $\mathrm{mg} \mathrm{mL}^{-1}$

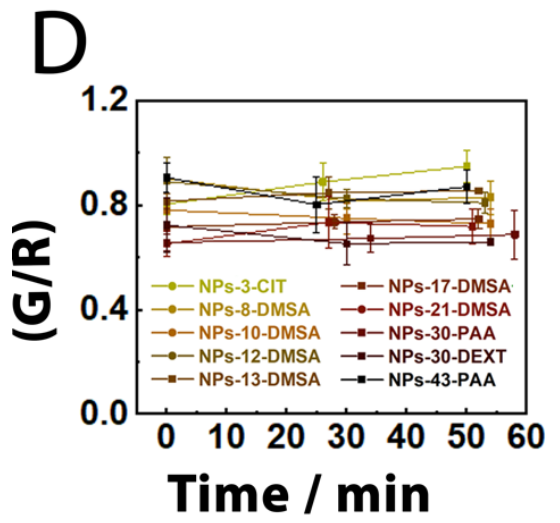




\section{WILEY-VCH}

Figure 3. (A) Mean and standard deviation values (SD) of each R, G and B values calculated from three measurement repetitions of the same solution and from a single measurement of three different solutions of the same concentration. (B) G/R values at different concentrations of sample NPs- NPs-30-PAA acquired in different days. (C) G/R values at different concentrations of samples NPs-10-DMSA and NPs-30-PAA determined from images taken with two different smartphones. Smartphone one is a Samsung J3 and smartphone 2 is a Xiaomi A2. (D) G/R data of all the samples acquired repeated times along $1 \mathrm{~h}$ to verify the stability of the sample.

The linear relationship of the $G / R$ ratio with the iron concentration of the nanoparticle suspensions, that only occurs at a given concentration range, opens up the possibility of using this procedure to calculate the iron concentration of a suspension of nanoparticles from the RGB colors acquired with the smartphone camera through the interpolation in a previously determined calibration curve (Figure 4A). 

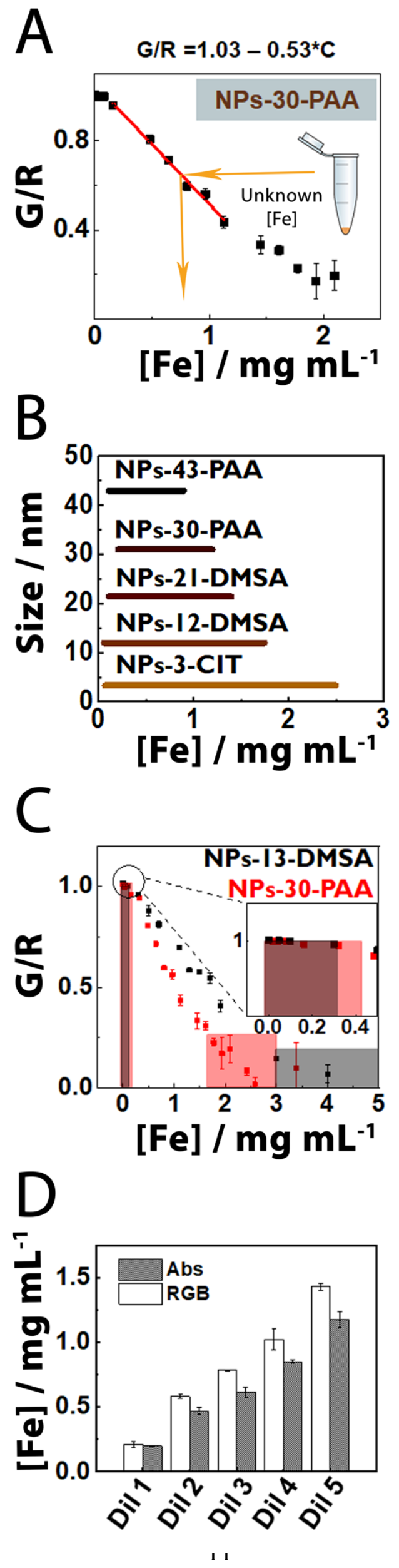


\section{WILEY-VCH}

Figure 4. (A) Schematic representation of the quantification of the iron concentration from samples with unknown iron content using a calibration curve based on G/R data previously shown in Figure 2. (B) Concentration range in which G/R data displays a linear behavior with the iron concentration for selected nanoparticles. (C) $G / R$ values obtained at different concentrations for samples NPs-13-DMSA and NPs-30- PAA. The grey and red areas indicate the regions in which $\mathrm{G} / \mathrm{R}$ does not linearly depend on the iron concentration for both samples. (D) Iron content determined by both the smartphone-based colorimetric method (RGB) and the spectroscopic one (Abs) for several dilutions of sample NPs-30-PAA.

The range in which a linear correlation of the $\mathrm{G} / \mathrm{R}$ ratio with the iron concentration is maintained is different for each material (Figure 4B). That implies that the range in which this method could be used would be slightly different for each kind of particles, being the lower limits around $\approx 0.1-0.2 \mathrm{mg} \mathrm{Fe} \mathrm{mL}-1$ and the highest concentrations that could be measured before the color saturation in the range between 1 and $3 \mathrm{mg} \mathrm{Fe} \mathrm{mL}^{-1}$ (Figure 4B). Smaller samples, with the less negative slopes, display a broader concentration range in which the G/R ratio linearly varies with the iron concentration (Figure 4C). In contrast, bigger particles, have a shorter concentration range in which the $\mathrm{G} / \mathrm{R}$ ratio linearly varies with the concentration (Figure $4 \mathrm{C}$ ). It should be mentioned here that the detection limit obtained by this approach is worse than that obtained using more complex measurement techniques (e.g down to $1 \mathrm{ppm}$ Fe for inductively coupled plasma atomic emission spectroscopy (ICP-AES) $\left.{ }^{[20]}\right)$.

In order to verify the validity of the proposed smartphone-based colorimetric method, this approach has been tested using several dilutions of sample NPs-30-PAA, within the linear G/R vs $[\mathrm{Fe}]$ range. The iron content calculated from the RGB data has been calculated and compared with the iron content determined by our routine spectrophotometric method (Figure 4D). Values measured by the RGB method tend to provide slightly higher values of iron concentration than 


\section{WILEY-VCH}

the spectrophotometric method (Figure 4 D) and further analysis would be necessary to try to explain this behavior.

In spite of the higher detection limit and the lower accuracy of the RGB method in comparison with the commonly stablished ones, this method presents several important advantages. For a known material, it presents a very fast, simple, cheap and portable method to measure the iron content. In addition, it does not need any digestion step before the analysis, nor any complex equipment such as in the case of ICP-AES ${ }^{[20]}$ and similar techniques. It also does not need any special reagents as the ones used in quantification methods based on the formation of colored complexes of iron ions. ${ }^{[21-22]}$ Therefore, this approach maybe very useful in routine sample analysis, or in the verification of concentration values after the manipulation of the suspensions.

\subsection{RGB data can be used to determine the nanoparticles size}

An interesting feature of the analysis of the concentration ranges in which a linear correlation has been found for the $G / R$ values is that their slope is not unique for all the tested materials (Figure 5A). Surprisingly, there is a clear effect of the particle size on the G/R vs [Fe] slope, having the smallest particles a slope of $\approx-0.2 \mathrm{~mL} \mathrm{mg} \mathrm{Fe}^{-1}$ and the biggest particles a slope of $\approx$ $-0.6 \mathrm{~mL} \mathrm{mg} \mathrm{Fe}{ }^{-1}$ (Figure 5A). 


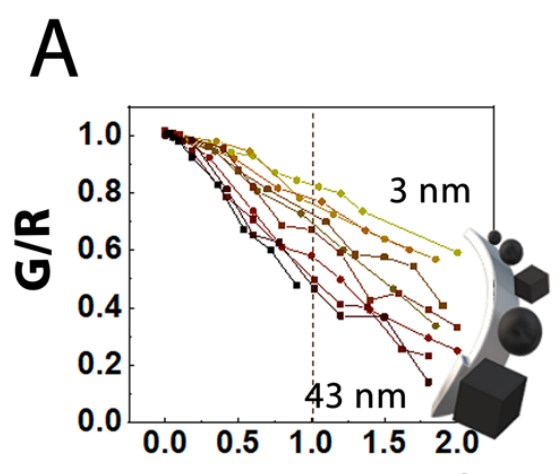

B

$[\mathrm{Fe}] / \mathrm{mg} \mathrm{mL}^{-1}$

$\longrightarrow-$ NPS-3-CIT

$\longrightarrow$ NPs-8-DMSA

$\longrightarrow$ NPs-10-DMSA

$\longrightarrow$ - NPs-12-DMSA

- NPs-13-DMSA

- - NPs-17-DMSA

$\longrightarrow$ NPs-21-DMSA

- - NPs-30-PAA

- NPs-30-DEXT

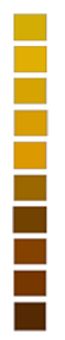

$[\mathrm{Fe}]=1 \mathrm{mg} \mathrm{mL}^{-1}$

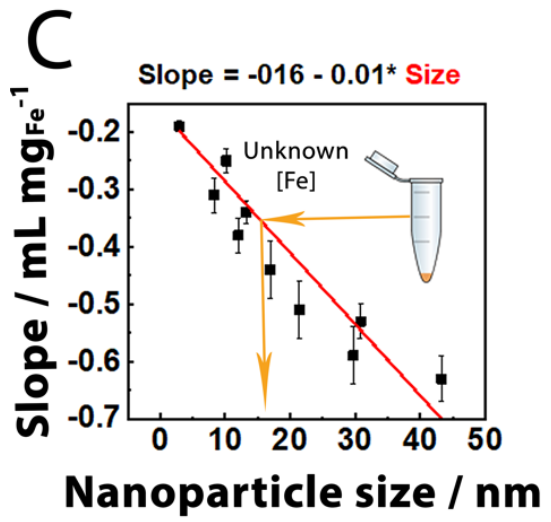

Figure 5. (A) G/R values at different iron concentrations for all the particles measured in this work. The color code is related with the names given in panel E. (B) List of the different particles characterized in this study and their corresponding color at a concentration of $1 \mathrm{mg} \mathrm{Fe}$ $\mathrm{mL}^{-1}$, marked with the dashed line in panel D. (C) Slope values from panel D for each of the different materials characterized in this work, plotted as a function of their corresponding size. This linear relationship could be used to estimate the nanoparticles size from suspensions with a known iron concentration. 


\section{WILEY-VCH}

This interesting phenomenon is the starting point for the development of a different method to get an estimation of the particle size from suspensions of particles using just the color captured by a smartphone from a suspension with a known iron concentration. In the same way as the different colors of a $\mathrm{pH}$ test strips help us to easily identify the $\mathrm{pH}$ of different solutions, the color of a suspension of nanoparticles at $1 \mathrm{mg} \mathrm{Fe} \mathrm{mL}^{-1}$ could be used to estimate their size just by taking a picture with the smartphone and comparing the color with our established color code (Figure 5B).

No clear effect of the particles coating has been found for the coating molecules used in this study. Particles with similar sizes but different coatings (NPs-30-DEXT and NPs-30-PAA) display a very similar slope. Nevertheless, it cannot be discarded that other coatings, such as the thick silica shells that sometimes are used to coat magnetic nanoparticles, ${ }^{[23]}$ have an impact on the color of the suspensions.

Also, no clear effect associated to the particle shape on the iron dependence of the G/R values has been found. Particles with similar size and different shapes (NPs-12-DMSA and NPs-13 DMSA) have an almost similar behavior. However, it cannot be discarded that other more complex shapes ${ }^{[24]}$ may present different behaviors.

In order to go one step further, the slopes of the G/R vs [Fe] data have been calculated for all the particles using the same concentration range $\left(0.5-1.5 \mathrm{mg} \mathrm{Fe} \mathrm{mL}^{-1}\right)$ (Figure $\mathrm{S} 2$ from the supporting information) and plotted as a function of the particle size determined from the transmission electron microscopy (TEM) images (Figure 5C). A linear relationship between these slopes and the nanoparticle size has been found. Therefore, more precise results could be achieved if, instead of taking a single picture of a suspension of $1 \mathrm{mg} \mathrm{Fe} \mathrm{mL}^{-1}$ sample, a dilution series is imaged and the slope of the G/R data vs [Fe] is calculated. In that case, the particles size could be obtained by using the Equation 1.

Size $=($ Slope +0.16$) /(-0.01)$ 


\section{WILEY-VCH}

There is a variety of methods that can be used to estimate the size of single core magnetic nanoparticles including TEM, dynamic light scattering (DLS) or magnetic measurements among others. ${ }^{[25]}$ All of them require expensive and non-portable equipment, time consuming protocols and sometimes the application of different models to finally extract the information regarding the particle size. Although the accuracy of the method proposed here is not the same as that obtained with these powerful techniques, this new alternative may be suitable for routine analysis of the production of different batches of particles, for example for very reproducible synthesis procedures. ${ }^{[26]}$ It may also be useful for smaller laboratories where the access to these techniques is limited. And it may also have an impact in remote areas, where magnetic nanoparticles are being used as part of biomedical applications, e.g. for the diagnosis of tropical diseases. ${ }^{[27]}$

This methodology could be extrapolated to other materials or suspending media. Nevertheless, some previous considerations should be kept in mind. In principle, the same procedure could be implemented to analyze suspensions of different materials, such as metallic nanoparticles (gold, silver, copper, etc) or oxides $\left(\mathrm{TiO}_{2}, \mathrm{ZnO}\right.$, etc) as long as they are stable in the suspending media. Nevertheless, a careful control on the NP aggregation should be performed over time, especially as a strong effect on the color has been described as a results of nanoparticle aggregation processes in some of these materials ${ }^{[28][29]}$. A suspending media different from water could also be employed, as long as the calibration curve is prepared using the same suspending media and that possible aggregation effects were discarded in advance. Another important parameter to consider would be the possible fast evaporation of the suspending media (e.g. in the case of organic solvents) that could lead to significant changes in the NP concentration in the suspension along the time-frame of the RGB data acquisition.

\section{Conclusion}




\section{WILEY-VCH}

A linear relationship between the $\mathrm{G} / \mathrm{R}$ ratio from iron oxide nanoparticle suspensions and their iron concentration has been found in the range between $\approx 0.1-0.2$ and $\approx 1-3 \mathrm{mg} \mathrm{Fe} \mathrm{mL}^{-1}$, depending on the particle size. This linear relationship is the starting point to use images acquired with the smartphone to quantify the iron content of suspensions containing such particles, resulting in a simple, fast, portable and low-cost method that could be easily implemented for routine tests. Moreover, a relationship between color and nanoparticle size has been found. The capacity of the color analysis to distinguish small color changes through the analysis of the RGB data has allowed the identification of this interesting relationship. This result has led to the development of another novel use of the color determination with the smartphone: the possibility of a fast and cheap method to determine the average size of iron oxide nanoparticles in suspension where the iron content is known. These two methods, have the potential to have a big impact in the materials research field, by expanding their use to nanoparticles with other compositions.

\section{Experimental Section}

Nanoparticle synthesis: Nanoparticles with $3 \mathrm{~nm}$ sizes were synthesized as previously described ${ }^{[26]}$. Briefly, a mixture of $\mathrm{FeCl}_{3} \cdot 6 \mathrm{H} 2 \mathrm{O}(75 \mathrm{mg}, 0.28 \mathrm{mmol})$ and citric acid trisodium salt $(80 \mathrm{mg}, 0.31 \mathrm{mmol})$ was dissolved in water $(9 \mathrm{~mL})$, after which hydrazine monohydrate (1 $\mathrm{mL}$ ) was added. The mixture was rapidly introduced into the microwave and heated at $240 \mathrm{~W}$ for $10 \mathrm{~min}$ with stirring at $120{ }^{\circ} \mathrm{C}$. After completion of this step, nanoparticles were purified through a gel filtration column (PD10) and stored in $5 \mathrm{~mL}$ vials for subsequent analysis.

Iron oxide magnetic nanoparticles with sizes in the range 9-21 nm, were synthesized by thermal decomposition of an iron oleate precursor in 1-octadecene in the presence of oleic acid. Particle size increased by decreasing the amount of oleic acid that slows down the growth step and by increasing the reaction time at reflux temperature. ${ }^{[30]}$ Particles were coated with dimercaptosuccinic acid (DMSA) by a ligand exchange process. The presence of $\mathrm{Na}$ impurities 
in the iron precursor seems to be responsible for the change in nanoparticle shape from spheres to cubes. ${ }^{[24]}$

Nanoparticles with sizes around 30-40 nm were synthesized by precipitation of an iron (II) salt $\left(\mathrm{FeSO}_{4}\right)$ in the presence of a base $(\mathrm{NaOH})$ and a mild oxidant $\left(\mathrm{KNO}_{3}\right)$. Reaction rate was controlled by the presence of ethanol in the media leading to a variation of the particle size from 43 to $30 \mathrm{~nm}$ when using a water/ethanol ratio equal to one. ${ }^{[31]}$ These particles were coated under high pressure homogenization conditions with dextran and poly(acrylic acid sodium salt). Finally, the particles were fractionated by controlled sedimentation.

Transmission Electron Microscopy (TEM): Samples were prepared by placing a drop of the diluted suspensions onto a carbon coated grid and allowing it to dry at room temperature. TEM studies were performed in a Tecnai G2 TEM (FEI) operated at $200 \mathrm{kV}$. The mean size of the iron oxide nanoparticles was determined by TEM analysis based on the manual measurement of over 200 individual nanoparticles using the public domain software ImageJ. The obtained histogram was fitted with a standard log-normal function.

RGB Data Acquisition: Two different smartphones were used: a Samsung J3 (Smartphone-1) and XiaoMi A2 Lite (Smartphone-2). In both devices, the rear camera was used for the image acquisition. The Smartphone 1 has a CMOS (Complementary metal-oxide-semiconductor) 8 MP sensor and $f / 2.2$ aperture, while the Smarphone 2 has Sony IMX486 Exmor RS camera with a 12 MP sensor and f/2.2 aperture. After the verification that both devices provided similar colors, Smartphone-2 was selected for the characterization of all the materials.

Different iron concentrations (between 0 and $4.5 \mathrm{mg} \mathrm{mL}^{-1}$, approximately) of the samples were prepared in 96-well plates. The wells contained $50 \mu \mathrm{l}$ of solution of each concentration. The solutions were photographed in a closed and dark cardboard box with an upper hole to place the camera. This set up allows maintaining the distance between the smartphone and the bottom 


\section{WILEY-VCH}

plates $(5 \mathrm{~cm})$ in all the measurements. The smartphone camera was placed on top of the box, facing parallelly to the well plate. Each solution was photographed in four points with help of the ColorGrab application (v 3.6.1 Loomatix Ltd.), and the color of each point was represented by RGB values. To maintain the light conditions, pictures were taken using the flashlight of the smartphone.

Iron concentration analysis: The concentration of iron in the nanoparticle sample suspensions was determined via a spectrophotometric method based on the determination of iron (III). ${ }^{[32]} \mathrm{A}$ fixed volume of NPs $(5 \mu \mathrm{L})$ was diluted in water $(45 \mu \mathrm{L})$. Then, aqua regia $\left(\mathrm{HCl} / \mathrm{HNO}_{3} 3: 1\right.$ ratio, $100 \mu \mathrm{L}$ ) were added to incubate all this mixture for $15 \mathrm{~min}$ at $60^{\circ} \mathrm{C}$. Afterwards, Milli-Q water $(350 \mu \mathrm{L})$ was added. Then, a fixed volume of this solution $(50 \mu \mathrm{L})$ was transferred to a 96-well plate. Apart, a solution containing a 5:1 ratio of $\mathrm{KOH}(4 \mathrm{~N})$ and 4,5-dihydroxy-1,3benzenedisulfonic acid (Tiron) was prepared and added $(60 \mu \mathrm{L})$ in each well, along with a $\mathrm{Na}_{3} \mathrm{PO}_{4}$ solution $(0.2 \mathrm{M}, \mathrm{pH}=9.7,100 \mu \mathrm{L})$. A final volume of $210 \mu \mathrm{L}$ was obtained and samples were allowed to stand at room temperature for $15 \mathrm{~min}$. Absorbance was measured at $480 \mathrm{~nm}$ on a spectrophotometer (Thermo Scientific, Multiskan GO). To get a calibration curve and estimate the concentration of iron(III) for the studied NPs, this method also employed six standard solutions of iron(III) with known concentrations of $0,0.1,0.2,0.4,0.6$ and $0.8 \mathrm{mg} \mathrm{Fe}$ $\mathrm{mL}^{-1}$ to measure absorbance and calculate the best fit line.

Statistical analysis: The arithmetic means and standard deviation (SD) values of each R, G and B values were calculated either from three measurement repetitions of the same solution or from a single measurement of three different solutions of the same concentration.

\section{Supporting Information}

Supporting Information is available from the Wiley Online Library or from the author. 


\section{WILEY-VCH}

\section{Acknowledgements}

This work was funded by European Commission (project NoCanTher, H2020, GA 685795), Spanish MINECO (SAF2016-79593-P to F.H., MAT2017-88148R (AEI/FEDER, UE) to M.P.M., MAT2015-71806-R to G.S. and PGC2018-096016-B-I00 to L.G.), and Fondo Social de la DGA (grupos DGA). Y.F-A. thanks Santander-Universidad Zaragoza Fellowship program for her PhD position. L.G. acknowledges financial support from the Ramón y Cajal program (RYC-2014-15512). G.S. acknowledges technical support from Dr. Leonor de la Cueva and Rebeca Amaro. Authors would like to acknowledge the use the Advanced Microscopy Laboratory (INA-Universidad de Zaragoza).

Received: ((will be filled in by the editorial staff))

Revised: ((will be filled in by the editorial staff)) Published online: ((will be filled in by the editorial staff))

\section{References}

[1] J. Starr, C. M. Schweik, N. Bush, L. Fletcher, J. Finn, J. Fish and C. T. Bargeron, PLoS One, 2014, 9, e111433.

[2] H. Wang, Y. J. Li, J. F. Wei, J. R. Xu, Y. H. Wang and G. X. Zheng, Anal. Bioanal. Chem., 2014, 406, 2799.

[3] S. Levin, S. Krishnan, S. Rajkumar, N. Halery and P. Balkunde, Sci. Total Environ., 2016, 551-552, 101.

[4] L. Shen, J. A. Hagen and I. Papautsky, Lab Chip, 2012, 12, 4240.

[5] J. Il Hong and B. Y. Chang, Lab Chip, 2014, 14, 1725.

[6] V. Oncescu, M. Mancuso and D. Erickson, Lab Chip, 2014, 14, 759.

[7] K. E. McCracken and J. Y. Yoon, Anal. Methods, 2016, 8, 6591.

[8] K. Su, Q. Zou, N. Hu and P. Wang, Sensors Actuators B Chem., 2015, 216, 134.

[9] M. Colombo, S. Carregal-Romero, M. F. Casula, L. Gutierrez, M. P. Morales, I. B. Bohm, J. T. Heverhagen, D. Prosperi and W. J. Parak, Chem. Soc. Rev., 2012, 41, 4306.

[10] R. M. Cornell and U. Schwertmann, The iron oxides : structure, properties, reactions, occurrences, and uses, Wiley-VCH, 2003. 


\section{WILEY-VCH}

[11] Jing Tang, M. Myers, K. A. Bosnick and L. E. Brus, J. Phys. Chem. B, 2003, 107, 7501.

[12] R. M. Cornell and U. Schwertmann, iron oxides Struct. Prop. React. Occur. uses.

[13] A. Espinosa, J. Kolosnjaj - Tabi, A. Abou - Hassan, A. Plan Sangnier, A. Curcio, A. K. A. Silva, R. Di Corato, S. Neveu, T. Pellegrino and L. M. Liz - Marzán, Adv. Funct. Mater., 2018, 28, 1803660.

[14] D. Li, W. Y. Teoh, J. J. Gooding, C. Selomulya and R. Amal, Adv. Funct. Mater., 2010, 20, 1767.

[15] U. O. Häfeli, J. S. Riffle, L. Harris-Shekhawat, A. Carmichael-Baranauskas, F. Mark, J. P. Dailey and D. Bardenstein, Mol. Pharm., 2009, 6, 1417.

[16] R. Mejías, P. Hernández Flores, M. Talelli, J. L. Tajada-Herráiz, M. E. F. Brollo, Y. Portilla, M. P. Morales and D. F. Barber, ACS Appl. Mater. Interfaces, 2019, 11, 340.

[17] B. D. Fellows, N. Ghobrial, E. Mappus, A. Hargett, M. Bolding, D. Dean and O. T. Mefford, Nanomedicine Nanotechnology, Biol. Med., 2018, 14, 1191.

[18] M. Hedayati, B. Abubaker-Sharif, M. Khattab, A. Razavi, I. Mohammed, A. Nejad, M. Wabler, H. Zhou, J. Mihalic, C. Gruettner, T. DeWeese and R. Ivkov, Int. J. Hyperth., 2018, 34, 373.

[19] J. Guo, J. X. H. Wong, C. Cui, X. Li and H. Z. Yu, Analyst, 2015, 140, 5518.

[20] R. Costo, D. Heinke, C. Grüttner, F. Westphal, M. P. Morales, S. VeintemillasVerdaguer and N. Gehrke, Anal. Bioanal. Chem., 2019, 411, 1895.

[21] J. H. Yoe and A. L. Jones, Ind. Eng. Chem. - Anal. Ed., 1944, 16, 111.

[22] S. Boutry, D. Forge, C. Burtea, I. Mahieu, O. Murariu, S. Laurent, L. Vander Elst and R. N. Muller, Contrast Media Mol. Imaging, 2009, 4, 299.

[23] M. Asgari, M. Soleymani, T. Miri and A. Barati, J. Mol. Liq., 2019, 292, 111367.

[24] A. G. Roca, L. Gutiérrez, H. Gavilán, M. E. Fortes Brollo, S. Veintemillas-Verdaguer and M. del P. Morales, Adv. Drug Deliv. Rev., 2019, 138, 68.

[25] F. Ludwig, C. Balceris, T. Viereck, O. Posth, U. Steinhoff, H. Gavilan, R. Costo, L. Zeng, E. Olsson, C. Jonasson and C. Johansson, J. Magn. Magn. Mater., 2017, 427, 19.

[26] J. Pellico, J. Ruiz-Cabello, I. Fernández-Barahona, L. Gutiérrez, A. V Lechuga-Vieco, J. A. Enríquez, M. P. Morales and F. Herranz, Langmuir, 2017, 33, 10239.

[27] C. G. Lindholz, V. Favero, C. de M. Verissimo, R. R. F. Candido, R. P. de Souza, R. R. dos Santos, A. L. Morassutti, H. R. Bittencourt, M. K. Jones, T. G. St. Pierre and C. Graeff-Teixeira, PLoS Negl. Trop. Dis., 2018, 12, 1.

[28] J. Nam, N. Won, H. Jin, H. Chung and S. Kim, J. Am. Chem. Soc., 2009, 131, 13639. 


\section{WILEY-VCH}

[29] S. D. Solomon, M. Bahadory, A. V. Jeyarajasingam, S. A. Rutkowsky, C. Boritz and L. Mulfinger, J. Chem. Educ., 2007, 84, 322.

[30] G. Salas, C. Casado, F. J. Teran, R. Miranda, C. J. Serna and M. P. Morales, J. Mater. Chem., 2012, 22, 21065.

[31] M. Andrés Vergés, R. Costo, A. G. Roca, J. F. Marco, G. F. Goya, C. J. Serna and M. P. Morales, J. Phys. D. Appl. Phys., 2008, 41, 134003.

[32] R. M. Fratila, M. Navascuez, J. Idiago-López, M. Eceiza, J. I. Miranda, J. M. Aizpurua and J. M. De La Fuente, New J. Chem., 2017, 41, 10835. 